\title{
RAMP UP DA MAIOR INSTALAÇÃO DE MOINHOS VERTIMILL DO MUNDO
}

\author{
D. B. MAZZINGHY*, H. G. D. TURRER, J. F. C. RUSSO e C. L. SCHNEIDER \\ Anglo American e Centro de Tecnologia Mineral \\ douglas.mazzinghy@angloamerican.com*
}

Artigo submetido em novembro/2015 e aceito em novembro/2015

DOI: $10.15628 /$ holos.2015.3664

\section{RESUMO}

O circuito de remoagem do projeto Minas-Rio é atualmente o maior do mundo utilizando moinhos verticais. O circuito possui 16 Vertimill VTM-1500 da Metso e 8 baterias de ciclones. 0 início das operações ocorreu no final de 2014 e os moinhos têm se mostrado bem robustos para a aplicação selecionada. 0 minério processado é um itabirito que é concentrado através de flotação reversa. O circuito de remoagem tem como objetivo reduzir a granulometria do concentrado final para facilitar o transporte através de um mineroduto com mais de $525 \mathrm{~km}$ de extensão. O presente trabalho apresenta os dados obtidos através de amostragens do circuito industrial além de testes em escala de laboratório para estimativa de consumo energético. Estes resultados também são comparados com os testes em escala de laboratório e piloto executados para dimensionamento do circuito de remoagem.

PALAVRAS-CHAVE: Remoagem, Vertimill, Minas-Rio, Itabiríto, Consumo energético.

\section{RAMP UP OF THE LARGEST VERTIMILL INSTALLATION IN THE WORLD}

\section{ABSTRACT}

The regrinding circuit of the Minas-Rio project is currently the world's largest using vertical mills. The circuit has 16 VTM-1500 Metso Vertimills and 8 cyclones clusters. The start-up occurred in late 2014 and the mills have been shown to be robust for the selected application. The ore processed is an Itabirite which is concentrated by reverse flotation. The target of the regrind circuit is to reduce the particle size distribution of the final concentrate to
\end{abstract}

facilitate pumping through a pipeline of more than 525 $\mathrm{km}$ long. This paper presents the data obtained from the industrial circuit sampling as well as tests on a laboratory scale used to estimate the energy consumption. These results are also compared with tests in laboratory and pilot scale carried out for sizing the regrind circuit.

KEYWORDS: Regrind, Vertimill, Minas-Rio, Itabirite, Energy consumption. 


\section{INTRODUÇÃO}

O Minas-Rio, de propriedade da Anglo American, é um complexo concebido para produzir 24,5 milhões de tonelada por ano de Pellet Feed (base seca). O sistema engloba mina, planta, mineroduto, filtragem, estocagem e porto. A Figura 1 mostra todas as instalações do sistema Minas-Rio.

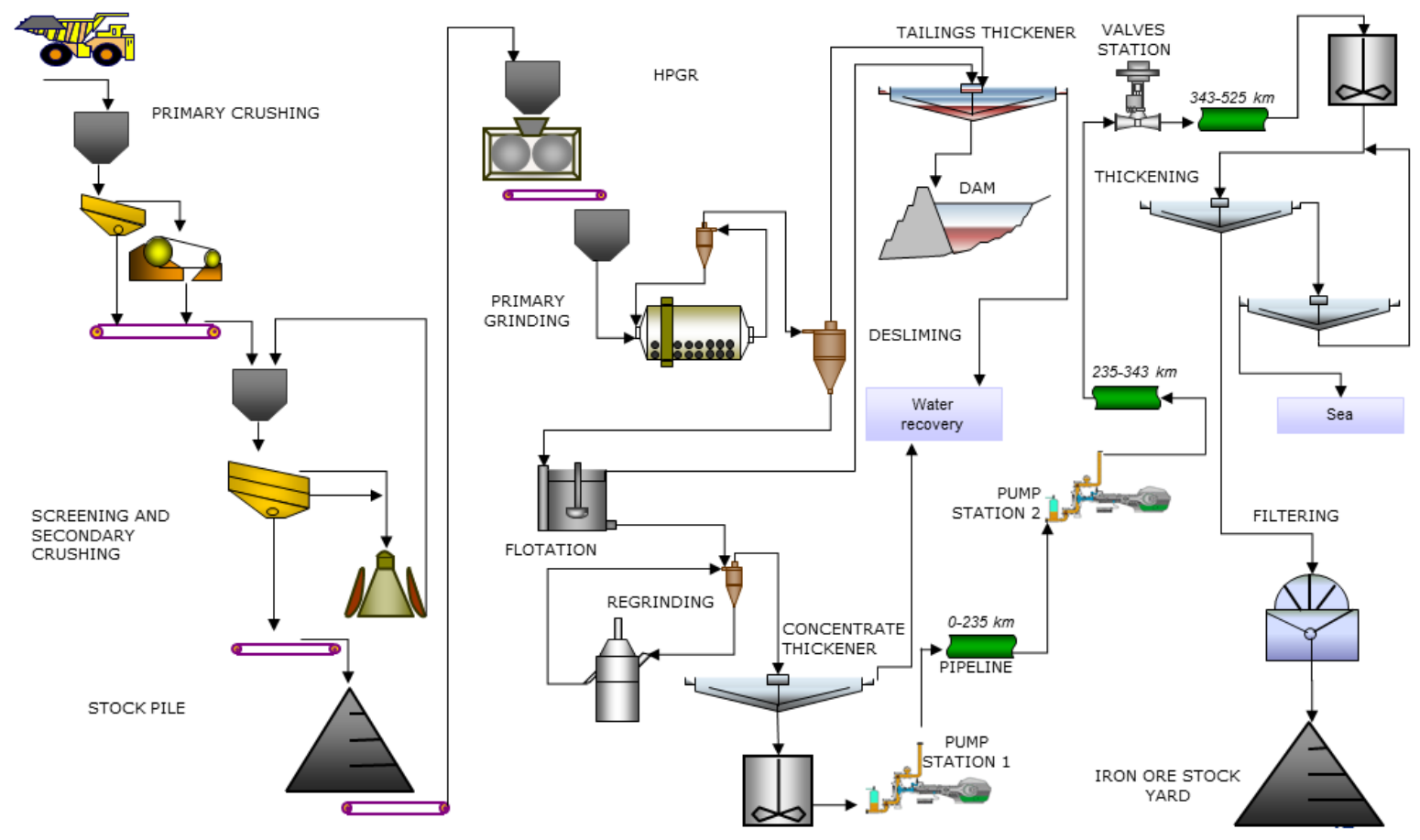

Figura 1: Sistema Minas-Rio

A mina e a planta estão localizadas na cidade de Conceição do Mato Dentro em Minas Gerais. O mineroduto é o maior do mundo com 529 km de extensão ligando a planta ao porto no Rio de Janeiro.

Atualmente existem mais de 420 moinhos Vertimill instalados no mundo totalizando cerca de $300 \mathrm{MW}$ de potência (Merriam et al., 2015). O circuito de remoagem do Minas-Rio é o maior do mundo a utilizar moinhos verticais. Os moinhos foram fornecidos pela Metso e são do modelo VTM-1500. A potência de cada Vertimill é de 1119 kW. São 16 moinhos instalados, totalizando 17,9 MW de potência. A remoagem do Minas-Rio está dividida em 2 prédios, sendo cada um deles equipado com 8 Vertimill e 4 baterias de ciclones. Cada bateria de ciclones possui 8 ciclones instalados, totalizando 64 ciclones de $500 \mathrm{~mm}$ de diâmetro. Cada prédio é alimentado através de um tanque com agitador que recebe o concentrado obtido através da etapa de flotação.

$\mathrm{O}$ circuito de remoagem tem como objetivo reduzir o material até um $\mathrm{P} 80=36 \mu \mathrm{m}$ para alimentação do mineroduto que transporta o Pellet Feed da cidade de Conceição do Mato Dentro no estado de Minas Gerais até o porto Açu na cidade de São João da Barra no estado do Rio de Janeiro. 
O projeto está na fase de ramp up e até o momento o circuito de remoagem não apresentou maiores problemas. A Figura 2 mostra o circuito de remoagem do Minas-Rio.

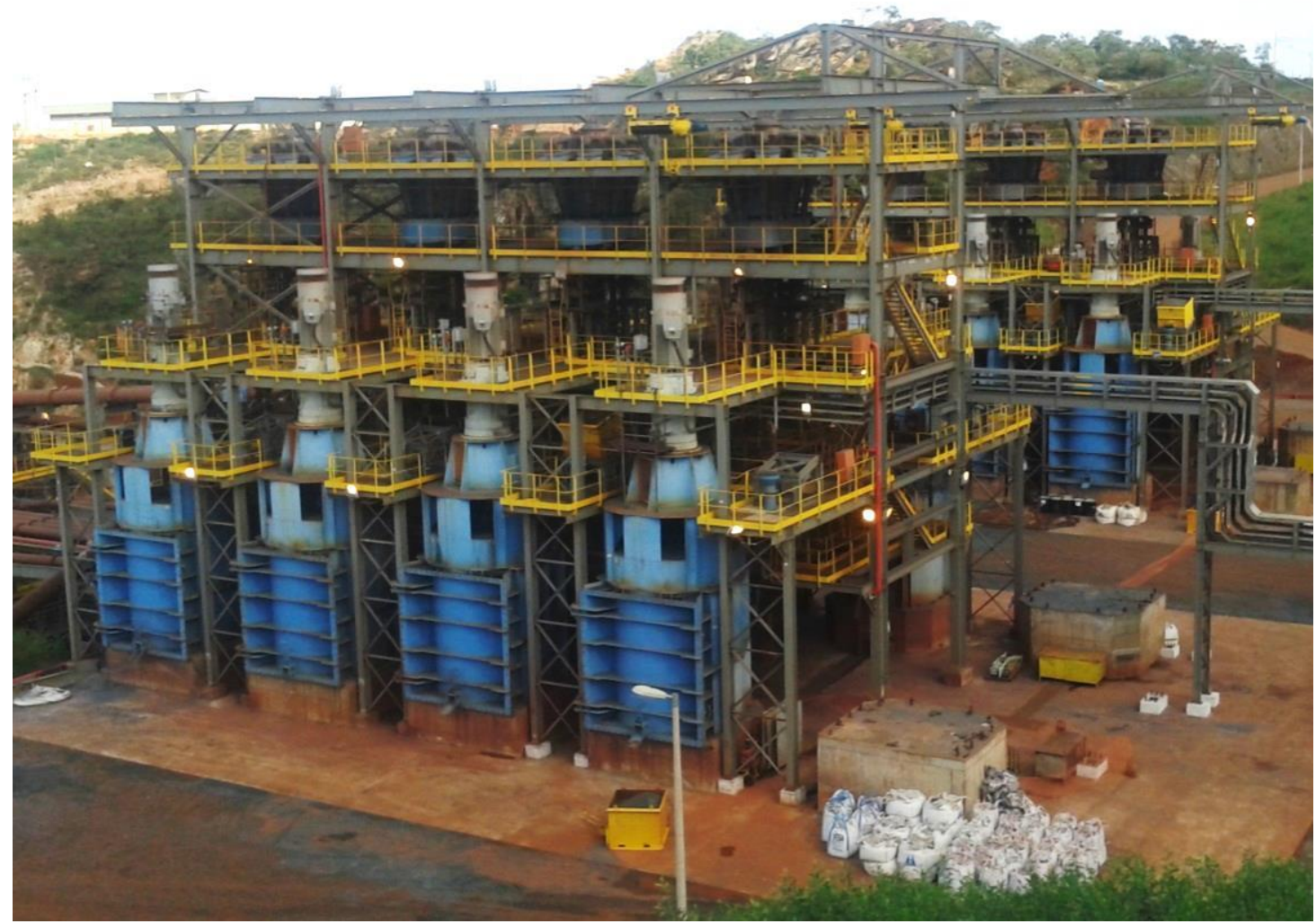

Figura 2: Circuito de remoagem do Minas-Rio

O presente trabalho tem como objetivo: apresentar e discutir os dados obtidos através de amostragens do circuito de remoagem; comparar o consumo energético obtido industrialmente com o consumo energético obtidos nos testes em escala de laboratório executados conforme a metodologia proposta pela Metso, fabricante dos moinhos Vertimill; apresentar os resultados dos testes em escala de laboratório e piloto executados para dimensionado do circuito de remoagem.

\section{MATERIAIS E MÉTODOS}

\subsection{Amostragem}

Uma campanha de amostragem foi realizada no circuito de remoagem do Projeto MinasRio. Amostras de todos os fluxos do circuito foram coletadas para a determinação da distribuição granulométrica, concentração de sólidos e peso específico. Os dados de potência e taxa de alimentação foram obtidos através do sistema de controle da planta.

A Figura 3 apresenta o fluxograma do circuito de remoagem do Minas-Rio. 
Pontos de Amostragem

Bateria de Ciclones 8 Ciclones de $500 \mathrm{~mm}$

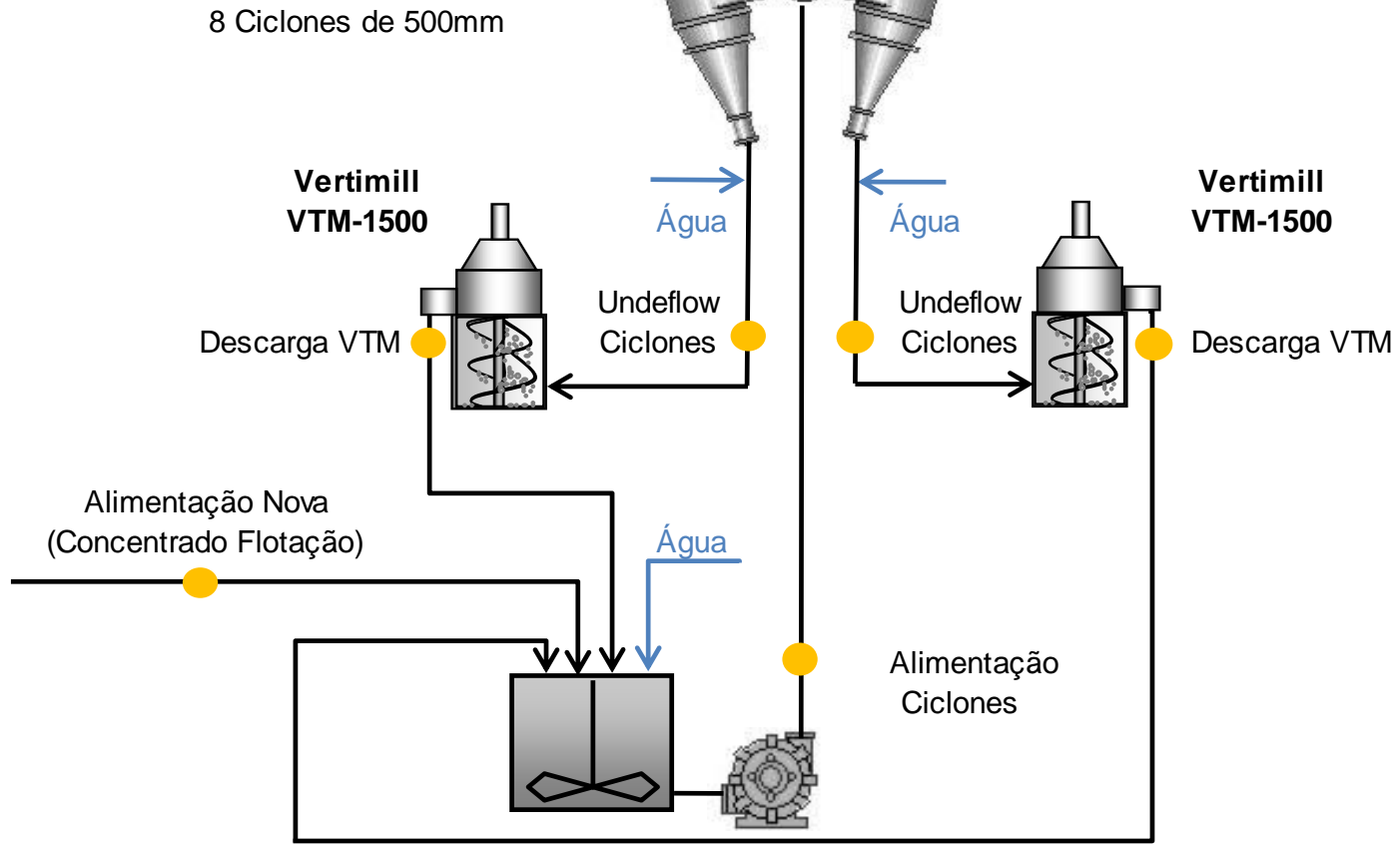

Figura 3: Fluxograma do circuito de remoagem do Minas-Rio

\subsection{Balanço de Massas}

O balanço de massas foi elaborado através do método de Lagrange (Valadão \& Araujo, 2007) implementado em planilhas de Excel $^{\circledR}$ utilizando os dados obtidos na amostragem e pelo sistema de controle.

\subsection{Testes de Moagem em Escala de Laboratório}

Testes de bancada foram realizados com amostras da alimentação nova do circuito de remoagem. Foi utilizado um moinho tubular convencional. $O$ teste seguiu as mesmas premissas do teste normalmente realizado pela Metso para estimativa de consumo energético do Vertimill.

As amostras foram moídas em três intervalos de tempo fixados em 20, 40 e 60 minutos. Após o último tempo de moagem obteve-se um P80 de aproximadamente $36 \mu \mathrm{m}$. Conhecendo a potência demanda no teste em $\mathrm{kW}$ e a massa de sólidos contida no moinho em $\mathrm{t}$, calcula-se o consumo específico de energia em $\mathrm{kWh} / \mathrm{t}$. Este valor é então multiplicado por um fator de 0,65 para se obter a estimativa de consumo energético do circuito de remoagem industrial com Vertimill.

A Tabela 1 mostra os parâmetros operacionais do teste chamado de Jar Mill Test, ou teste de jarro (Metso, 2011). 
Tabela 1: Parâmetros do teste de bancada com moinho tubular

\begin{tabular}{cc}
\hline Parâmetros & Valores \\
\hline Diâmetro (m) & 0,208 \\
Comprimento (m) & 0,208 \\
Porosidade (\%) & 40 \\
J - Enchimento Bolas (\%) & 42 \\
U - Enchimento Material (\%) & 100 \\
Velocidade Crítica (\%) & 76 \\
\hline
\end{tabular}

\section{RESULTADOS E DISCUSSÃO}

\subsection{Balanço de Massas}

As Figuras de 4 a 8 mostram os resultados de quatro amostragens realizadas no circuito de remoagem. Os pontos indicam os dados experimentais e a linha indica a média obtida através dos dados experimentais.

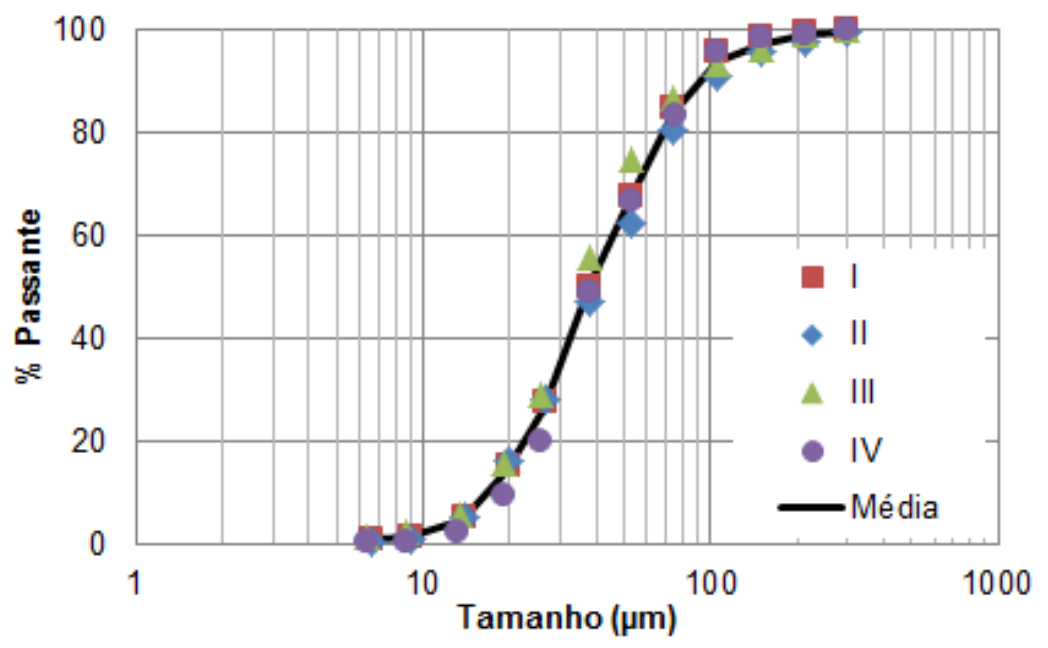

Figura 4: Amostragem da alimentação nova do circuito

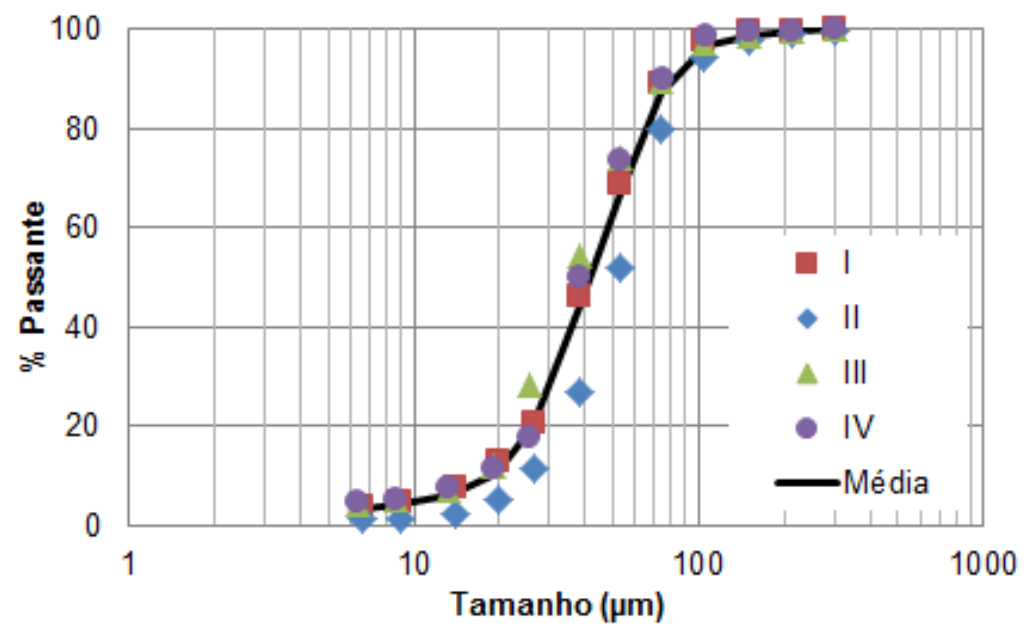

Figura 5: Amostragem da descarga do moinho 


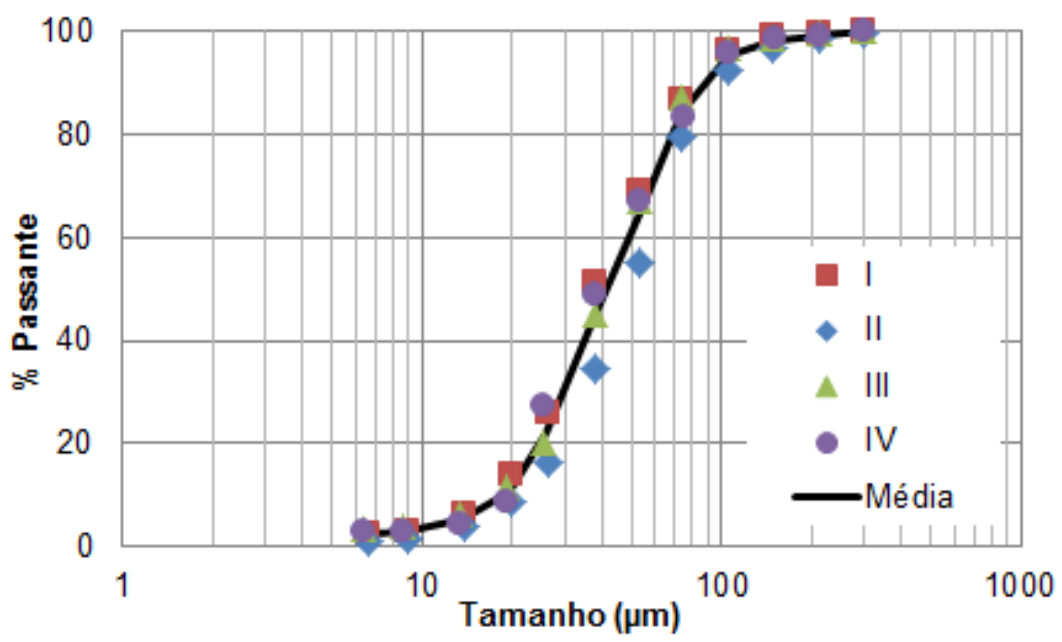

Figura 6: Amostragem da alimentação da bateria de ciclones

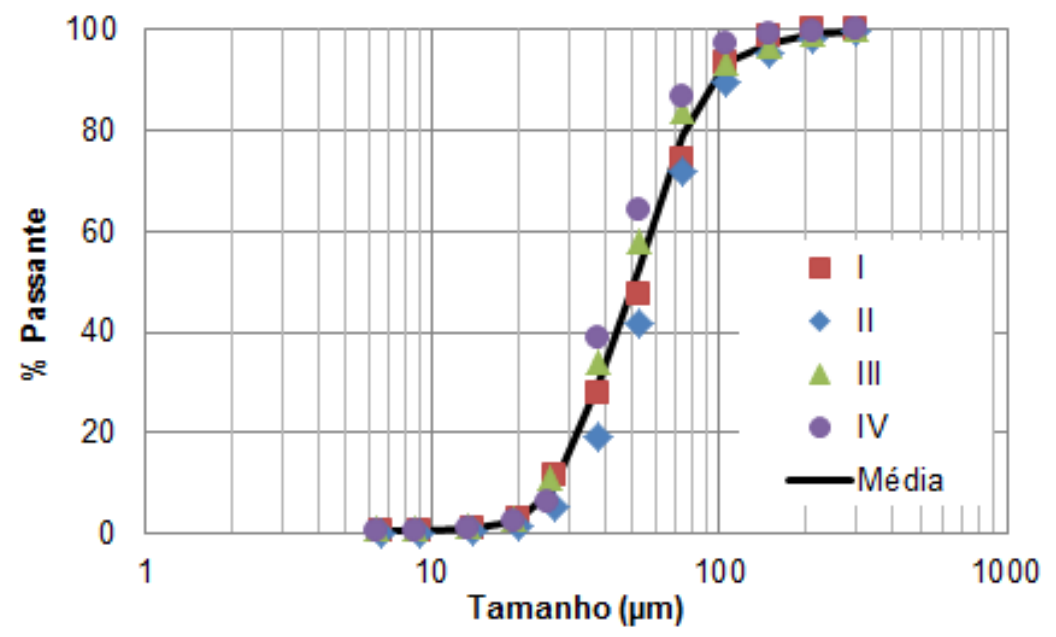

Figura 7: Amostragem do undeflow da bateria de ciclones

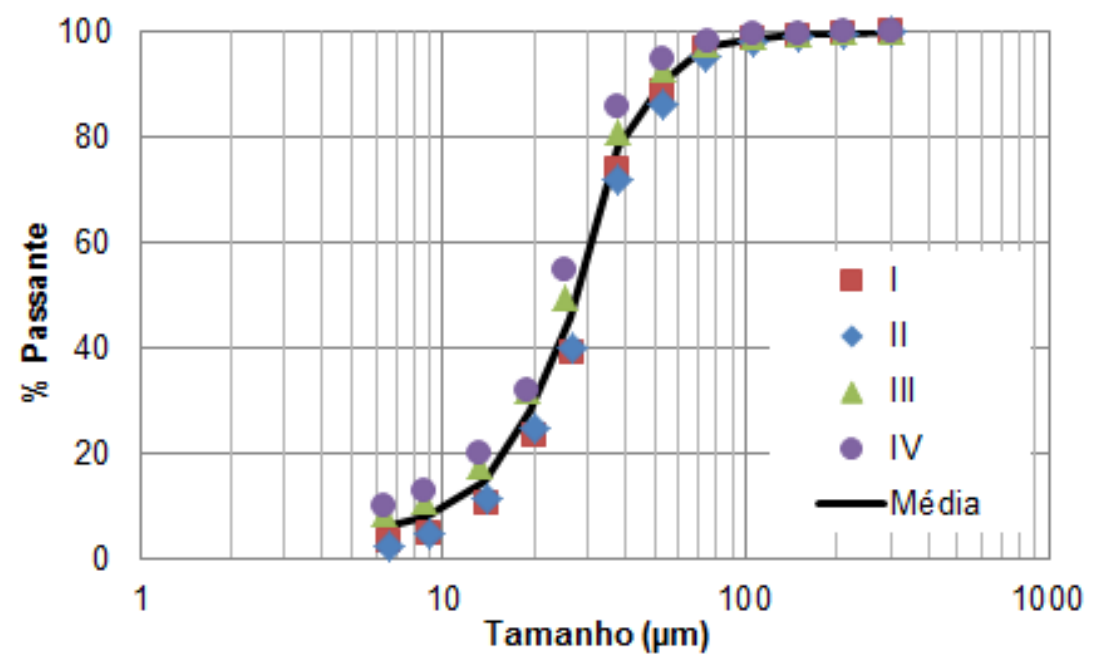

Figura 8: Amostragem do overflow da bateria de ciclones 
Percebe-se que os formatos das curvas granulométricas são similares em todas as amostragens. A operação está na fase de ajustes dos equipamentos e por isso o produto do circuito de remoagem (overflow dos ciclones) ainda apresenta variação (Figura 8).

A Figura 9 mostra a média de todas as amostragens.

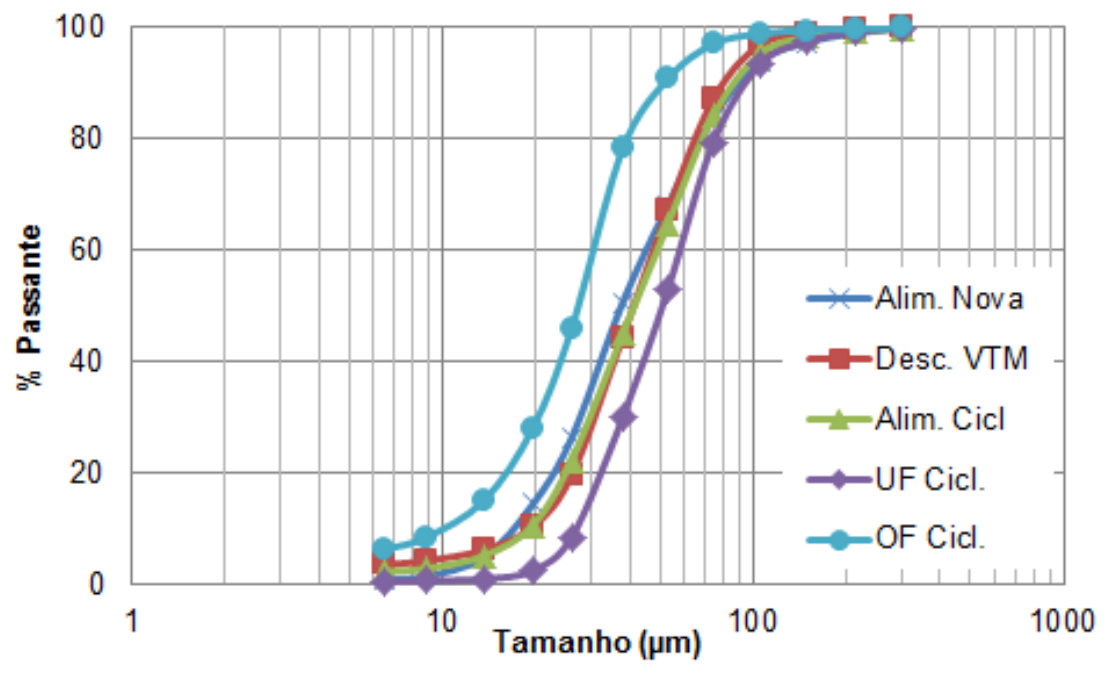

Figura 9: Média de todos os fluxos amostrados

\subsection{Testes de Moagem em Escala de Laboratório}

A Figura 10 mostra os resultados do teste executado com um moinho tubular em escala de laboratório utilizando as mesmas condições operacionais adotadas pela Metso para estimativa de consumo energético de moinho Vertimill em escala industrial.

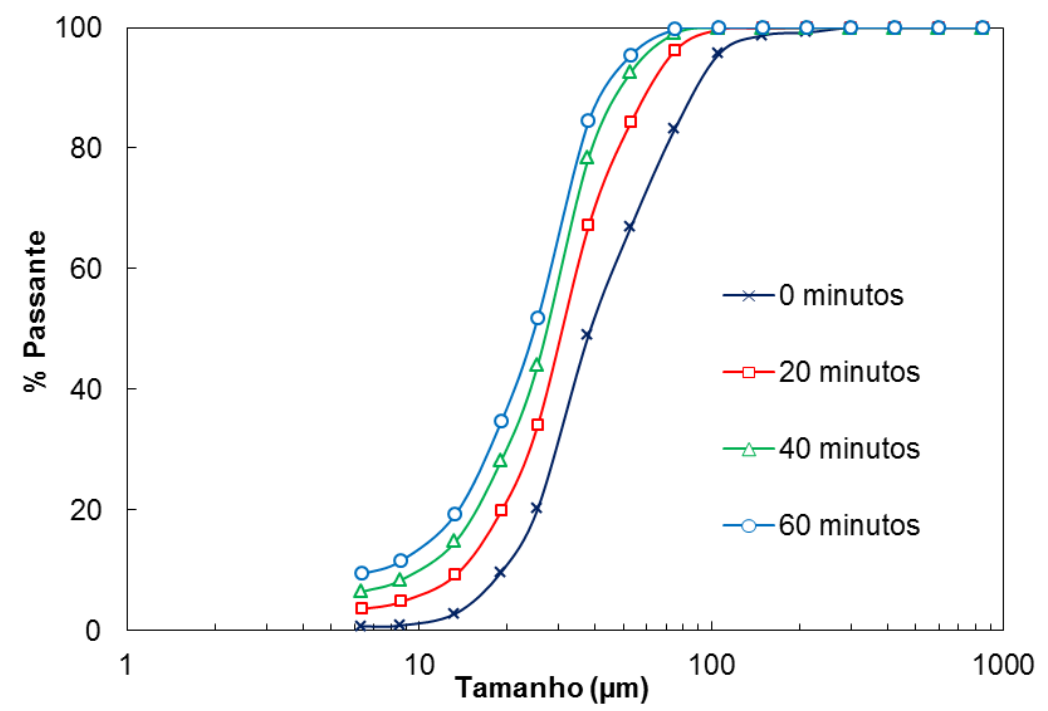

Figura 10: Curvas granulométricas obtidas no teste de moagem em laboratório

$\mathrm{O}$ Jar Test executado resultou em $8,9 \mathrm{kWh} / \mathrm{t}$ para atingir um P80 $=36 \mu \mathrm{m}$. Este consumo energético foi multiplicado pelo fator de 0,65 adotado pela Metso, resultando em uma estimativa de consumo energético para o Vertimill em escala industrial igual a 5,8 kWh/t.

Consumo Energético em Escala de Laboratório, Piloto e Industrial 
A Tabela 2 apresenta os dados utilizados no dimensionamento do circuito de remoagem (Metso, 2011) e os dados obtidos industrialmente.

Tabela 2: Consumo Energético Laboratório, Piloto e Industrial

\begin{tabular}{ccccc}
\hline Período & \multicolumn{2}{c}{ Projeto } & \multicolumn{2}{c}{ Atual } \\
\hline Dados & Lab. & Piloto & Lab. & Industrial \\
\hline$F_{80}(\mu \mathrm{m})$ & 67 & 67 & 62 & 62 \\
$P_{80}(\mu \mathrm{m})$ & 36 & 37 & 36 & 35 \\
Energia Específica $(\mathrm{kWh} / \mathrm{t})$ & 6,5 & 5,3 & 5,8 & 5,6 \\
\hline
\end{tabular}

Todos os dados de energia específica foram calculados considerando a potência líquida de moagem. A potência líquida do Vertimill em escala industrial foi calculada considerando que $10 \%$ da potência bruta se refere a potência em vazio.

Os resultados dos testes em escala de laboratório e piloto executados para definição do circuito de remoagem estão bem coerentes com os resultados encontrados nas amostragens do circuito industrial.

\section{CONCLUSÕES}

A maior instalação de moinhos Vertimill do mundo tem sido operada sem maiores problemas até o momento e tem atingido a especificação de produto necessária para as etapas seguintes de processo. Considera-se que o circuito de remoagem do Minas-Rio tem boas oportunidades de ganhos através de otimização durante e após o fim da etapa de ramp up.

\section{AGRADECIMENTOS}

Agradecimento a Anglo American por permitir a divulgação dos dados e por incentivar a pesquisa constante para melhoria de suas operações, em especial aos colegas Valdiney Chaves, Luis Machado, Paula Pereira, Carlos Nascimento, Julio Silva e Fabiano Gonzaga.

\section{REFERÊNCIAS BIBLIOGRÁFICAS}

1. Merriam, K., Rotzinger, R., DeHart, I., Radziszewski, P., McDonnell, J., Assessing Vertimill Ultrafine Grinding Performance: The Gibraltar Mine Case, 47th Canadian Mineral Processors Conference, Ottawa, 2015.

2. Metso - Test Plant Report - Minas-Rio, Continuous VTM-3 Vertimill Test \& Special Jar Mill Grindability Test, August, 2011.

3. Valadão, G.E.S., Araujo, A.C. Introdução ao Tratamento de Minérios, Belo Horizonte, Editora UFMG, pp. 17-25, 2007. 